\title{
An Improved DMRS Design Scheme of LTE-Advanced Uplink
}

\author{
Xiaowen Li, Youbo Feng and Dan Wang \\ Key Laboratory of Mobile Communication Technology of Chongqing, \\ Chongqing University of Posts and Telecommunications, China \\ 520064564@qq.com
}

\begin{abstract}
The 3rd Generation Partnership Project (3GPP) Long Term Evolution (LTE) uplink supports only a single antenna transmission. However, in order to improve the uplink spectrum efficiency, support multiple antenna transmission in LTE-Advanced. Meanwhile, taking into account backwards compatibility for LTE. LTE-Advanced De-Modulation Reference Signal (DMRS) needs to be designed [1]. According to requirement of LTEAdvanced uplink pilot design, introducing orthogonal cover code (OCC) as a supplement, analysis and study of the current problems, proposes a Circular Shift (CS), Group/Sequence Hopping (GSH) and the OCC joint instructions method of pilot design. Finally, simulation and analysis of the performance of this scheme. Results show that this scheme can meet LTE-Advanced demand for pilot design well.
\end{abstract}

Keywords: LTE-Advanced, DMRS, Uplink, pilot design

\section{Introduction}

LTE-Advanced is a further evolution of LTE, aim to higher uplink and downlink peak rate, average throughput than LTE. Uplink MIMO is introduced in LTE-Advanced, including transmission diversity (TxD), single-user MIMO (SU-MIMO), multi-user MIMO (MU-MIMO), uplink supports a maximum of 4 transmit antennas. In order to support for uplink MIMO transmission in LTE-Advanced, DMRS and SRS need to be enhanced or modified [2]. This paper study about LTE-Advanced uplink DMRS designs.

Introduce orthogonal mask (OCC) as an aided orthogonal way of circular shift (CS) in LTE-Advanced uplink. OCC use Walsh codes at the length of 2. In order to using Walsh codes, the pilot symbols of two slots in a frame must be bound together, shown in Figure 1.

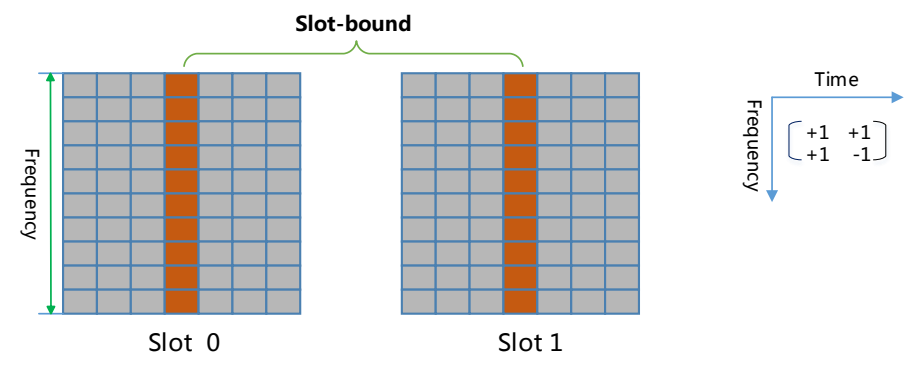

Figure 1. Pilot Slot-bound

As orthogonal of CS and supplement of OCC, there is a new design method of pilot, as shown in Figure 2 and Figure 3, respectively is 2-layer and 4-layer transmission pilot structure, using code division multiplexing (CDM), mainly include two DMRS structure: $\mathrm{CS}$ alone, CS and OCC combined.

- Pilot design supports 2-layer transport: 

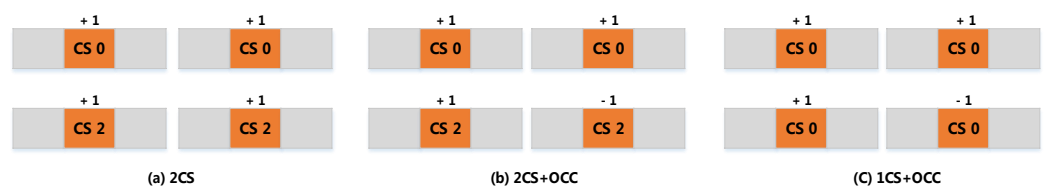

Figure 2. Different CS/OCC Structure Supports 2-layer Transport

Figure 2 (a) do not use OCC, only through a sequence of cyclic shifts to achieve orthogonal, is same with two antenna CDM design mode, each slot can estimate two antenna channel; Figure 2 (b) based on (a), combination of OCC to achieve orthogonal. Can jointly estimate two symbols, or separately estimate each signal, performance is better; Figure 2 (c) only use one sequence between layers, achieve orthogonal through OCC. two antenna channel cannot be estimated within a slot, to get channel characteristics, the pilot signal on the two moments must be combined to estimate.

- Pilot design supports 4-layer transport:
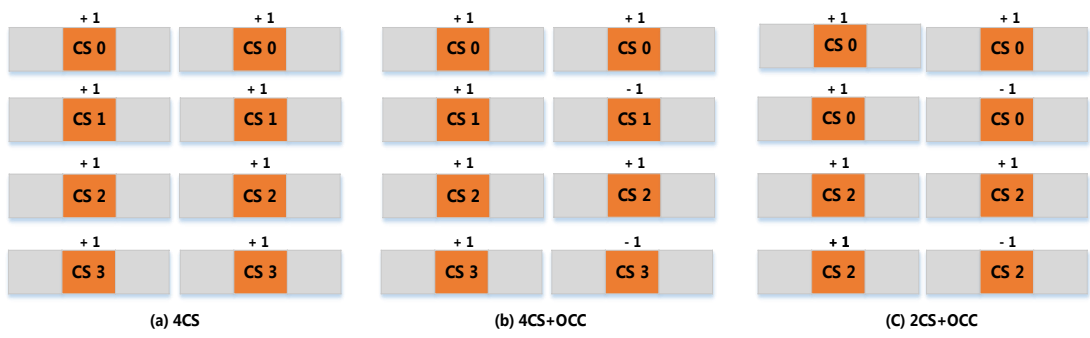

Figure 3. Different CS/OCC Structure Supports 4-layer Transport

Figure 3 (a) through a base sequence and its 3 cyclic shift sequences to achieve orthogonal of 4-layer, do not use OCC [3]. Figure 3 (b) based on (a), combination of OCC, can realize orthogonal very well between layers. Both of these methods are able to estimate 4-layer channel characteristics within a slot. Figure 3 (c) use only 2 pilot sequences, layer 1 and layer 2 use same base sequence CS1, through OCC to distinguish between the two, layer 3 and layer 4 use same cyclic shift sequence CS2, through the OCC to achieve orthogonal, unable to estimate the 4-layer channel in one slot, only combined two slots [4].

\section{Problems Existing in Design of LTE-Advanced DMRS}

To support SU-MIMO and unequally bandwidth matched MU-MIMO in LTEAdvanced, the pilot design face some problems. Introduce OCC is primarily intended to solve the problem of unequal bandwidth paired UE. Use OCC in $[1,1]$ or $[1,1]$, need instructions, cyclic shift sequence values for multiple antennas also require signal indicating, and how to use sequences hopping and group of sequences hopping in unequal bandwidth paired UE MU-MIMO [5]. The following are detailed analysis.

\subsection{The Cyclic Shift (CS) Value Indicator}

LTE-Advanced, request no new signaling in CS indicates, still use 3 bit DCI instructions, also hope to maintain good compatibility with LTE Rel-8. There are two solutions:

2.1.1. Fixed CS Offset Instructions: Same with LTE Rel-8, only indicates CS values $n_{D M R S}^{(2)}$ in reference layer (layer 0), other layers of the CS value are obtained by a predefined fixed CS offset, as shown in Table 1 (a). Assume have 4-layer, then layer 0 CS uses explicit signaling to indicate, other 3 layers CS of values obtained by CS 
offset fixed, and layer 2, 3, 4 CS offset values are 3, 6, 9 respectively. High-layer has good compatibility with lower-layer through this way. CS offset values of 3layer are $0,3,6,2$-layer are 0,6 . As far as possible to ensure margin maximization between layers.

2.1.2. Flexible CS Offset Instruction: Only indicates CS value in reference layer, CS value of other layers based on total number of transport layer, and as shown in Table 1 (b). For example, Assume have 3-layer, can obtain the interval between layers is 4 (12/3), other two CS offset values are 4 and 8 . This approach is able to guarantee the maximum spacing between layers, but need to know total number of layers, backward compatibility is not good.

Table 1. CS Offset Instructions

\begin{tabular}{|c|c|}
\multicolumn{2}{c}{ 2-layer } \\
\hline Layer & CS offset \\
\hline 0 & 0 \\
\hline 1 & 6 \\
\hline
\end{tabular}

\begin{tabular}{|c|c|}
\multicolumn{1}{c}{} & 3-layer \\
\hline Layer & CS offset \\
\hline 0 & 0 \\
\hline 1 & 6 \\
\hline 2 & 3 \\
\hline
\end{tabular}

\begin{tabular}{|c|c|}
\multicolumn{2}{c}{ 4-layer } \\
\hline Layer & CS offset \\
\hline 0 & 0 \\
\hline 1 & 6 \\
\hline 2 & 3 \\
\hline 3 & 9 \\
\hline
\end{tabular}

(a) Fixed CS offset

\begin{tabular}{|c|c|}
\multicolumn{2}{c}{ 2-layer } \\
\hline Layer & CS offset \\
\hline 0 & 0 \\
\hline 1 & 6 \\
\hline
\end{tabular}

\begin{tabular}{|c|c|}
\multicolumn{2}{c}{ 3-layer } \\
\hline Layer & CS offset \\
\hline 0 & 0 \\
\hline 1 & 4 \\
\hline 2 & 8 \\
\hline
\end{tabular}

\begin{tabular}{|c|c|}
\multicolumn{2}{c|}{ 4-layer } \\
\hline Layer & CS offset \\
\hline 0 & 0 \\
\hline 1 & 6 \\
\hline 2 & 3 \\
\hline 3 & 9 \\
\hline
\end{tabular}

(b) Flexible CS offset

\subsection{Orthogonal Cover Code (OCC) Signaling Indicator Method}

Introducing the OCC, unequally bandwidth UE multiplexing can be achieved, we can distinguish different UEs. But introducing the OCC would require signaling to indicate. Require instructions OCC does not increase the signaling overhead, and CS in small intervals use different values of OCC. Here are two methods of OCC's instructions [6].

2.2.1. Explicit Signaling Instruction: This uses 1 bit signaling to indicate what kind of OCC mode is used. Just need to CS indication signaling compression to 2 bit. Set 0 indicates use [1, 1], 1 indicates use [1, -1]. Signaling 000, the first bit indicates the OCC, the last two bit representing the CS offset value. This approach make available CS values reduce half.

2.2.2. Implicit Signaling Indication: According to CS values obtain implicitly what kind of OCC mode is used, as shown in Table 2. That is OCC instructions of 4-layer. This method requires no additional signaling and does not require CS signaling compression [7].

Table 2. OCC Implicit Signaling Indication

\begin{tabular}{|c|c|c|c|c|}
\hline & Layer 1 & Layer 2 & Layer 3 & Layer 4 \\
\hline CS & 0 & 3 & 6 & 9 \\
\hline OCC & {$[+1,+1]$} & {$[+1,-1]$} & {$[+1,+1]$} & {$[+1,-1]$} \\
\hline
\end{tabular}




\subsection{Group/Sequence Hopping (GSH) Instruction}

GSH can make inter-cell interference randomization, to achieve performance gains, Rel-8

GSH is a cell specific (Cell-specific), which means if close the GSH function, then all UEs in a cell is no longer supported by the GSH. So hopefully GSH in Rel-10 designed to be user-specific (UE-specific), that is, close or open GSH function only used for specific UE, Paired UEs in Rel-8 or 9 occupy the same resources, so it can support the inter slot GSH, if Rel-10 UE and Rel-8 or 9 UE pairs, paired UEs may be assigned different bandwidth resources, need to use OCC to realize orthogonal among UEs, so cannot use the inter slot GSH, But Rel-10 hopes to be able to continue using GSH to realize interference randomization [8]. So Rel-10 how to use GSH is a problem. Existing two solutions:

2.3.1. Keep the Rel-8 Mechanism: That is, when a different bandwidth allocation of UEs pairing, GSH function off, doing so will cause some loss of performance.

2.3.2. Using New Method of GSH: Sub-frames between GSH can meet above requires, but how to indicate is a problem, may increase the signaling overhead. Considering the existence of LTE-Advanced pilot design problem, a solution method is given below.

\section{An Improved DMRS Design Scheme}

\subsection{CS and OCC Design}

Rel-8 CS signaling indicates is determined primarily by $n_{D M R S}^{(2)}, n_{D M R S}^{(2)}$ valid values are 0 , $2,3,4,6,8,9,10$, with 3 bit said. Rel-10 CS design still used in this way, except for a small change, multiple antennas for a UE, simply indicates that the first antenna (layer 0) $n_{\text {DMRS }}^{(2)}$, By UL DCI format 3 bit cyclic shift indicator (cyclic shift indicator, CSI) to get, denoted by $n_{\mathrm{DMRS}, 0}^{(2)}$. Layer k CS value obtained by the formula $n_{\mathrm{DMRS}, \mathrm{k}}^{(2)}=\left(n_{\mathrm{DMRS}, 0}^{(2)}+\Delta_{k}\right) \bmod 2$, CS offset $\Delta_{k}$ changes with $\mathrm{k}$ value, When $\mathrm{k}=0,1, \Delta_{k}$ of 2-layer are 0,6 . When $\mathrm{k}=0,1$, $2,3, \Delta_{k}$ of 4-layer are $0,6,3,9$, When $\mathrm{k}=0,1,2, \Delta_{k}$ of 3-layer are $0,6,3$ or $0,8,4$. OCC indicates method combined with CS. According to the predetermined CS, alternating between layers [9], as shown in Table 3.1 and 3.2.

Table 3.1. OCC in DM-RS

\begin{tabular}{|c|c|}
\hline OCC Index: $I_{\text {occ }}$ & Orthoaonal Cover Code (OCC) \\
\hline 0 & {$[+1,+1]^{\mathrm{T}}$} \\
\hline 1 & {$[+1,-1]^{\mathrm{T}}$} \\
\hline
\end{tabular}

Table 3.2. CS and OCC in the Mapping of the DCI Format

\begin{tabular}{|c|c|c|c|}
\hline $\begin{array}{c}\text { Cyclic Shift Field } \\
\text { In DCI format 0 }\end{array}$ & $n_{D M R S}^{(2)}$ & $I_{o c c}$ & $n_{\text {DMRS }}^{O C C}$ \\
\hline 000 & 0 & 0 & {$[+1,+1]$} \\
\hline 001 & 6 & 1 & {$[+1,-1]$} \\
\hline 010 & 3 & 1 & {$[+1,-1]$} \\
\hline 011 & 4 & 0 & {$[+1,+1]$} \\
\hline 100 & 2 & 1 & {$[+1,-1]$} \\
\hline 101 & 8 & 0 & {$[+1,+1]$} \\
\hline
\end{tabular}




\begin{tabular}{|c|c|c|c|}
\hline 110 & 10 & 1 & {$[+1,-1]$} \\
\hline 111 & 9 & 0 & {$[+1,+1]$} \\
\hline
\end{tabular}

With the above definition, we can design different antenna transmission DMRS, following are given Rank-1 Rank-4 transmission of CS and OCC design.

Table 3.3. Rank-1 Transmission

\begin{tabular}{|c|c|}
\hline Layer (number of virtual antennas) & DM-RS in slot 0 and 1 \\
\hline 0 & CSO: $n_{\mathrm{DMRS}}^{(2)}$, OCC Index: $I_{\text {occ }}$ \\
\hline
\end{tabular}

Table 3.4. Rank-2 Transmission

\begin{tabular}{|c|c|}
\hline Layer (number of virtual antennas) & DM-RS in slot 0 and 1 \\
\hline 0 & CSO: $n_{\text {DMRS }}^{(2)}$ OCC Index: $I_{o c c}$ \\
\hline 1 & CS1: $n_{\text {DMRS }}^{(2)}+6$, OCC Index: $1-I_{o c c}$ \\
\hline
\end{tabular}

Table 3.5. Rank-3 Transmission

\begin{tabular}{|c|c|}
\hline Layer (number of virtual antennas) & DM-RS in slot 0 and 1 \\
\hline 0 & CSO: $n_{\mathrm{DMRS}}^{(2)}$, OCC Index: $I_{o c c}$ \\
\hline 1 & $\mathrm{CS} 1: n_{\mathrm{DMRS}}^{(2)}+3$, OCC Index: $1-I_{o c c}$ \\
\hline 2 & $\mathrm{CS} 2: n_{\mathrm{DMRS}}^{(2)}+6$, OCC Index: $I_{o c c}$ \\
\hline
\end{tabular}

Table 3.6. Rank-4 Transmission

\begin{tabular}{|c|c|}
\hline Layer (number of virtual antennas) & DM-RS in slot 0 and 1 \\
\hline 0 & CSO: $n_{\mathrm{DMRS}}^{(2)}$, OCC Index: $I_{\text {occ }}$ \\
\hline 1 & $\mathrm{CS} 1: n_{\mathrm{DMRS}}^{(2)}$, OCC Index: $1-I_{o c c}$ \\
\hline 2 & $\mathrm{CS} 2: n_{\mathrm{DMRS}}^{(2)}+6$, OCC Index: $I_{o c c}$ \\
\hline 3 & $\mathrm{CS} 3: n_{\mathrm{DMRS}}^{(2)}+9$, OCC Index: $1-I_{o c c}$ \\
\hline
\end{tabular}

$n_{D M R S}^{(2)}$ and $I_{\text {occ }}$ can get from Table 3.2. Value is $0,2,3,4,6,8,9,10$, use 3 bit representation $(000, \ldots, 111)$, get $n_{D M R S}^{(2)}$ value from Table 3.2 and get OCC values corresponding, $I_{\text {occ }}=0$ means $[1,1], I_{\text {occ }}=1$ means $[1,-1]$. Does not require a specific signaling description, other layers of CS and OCC get from Table 3.3 Table 3.6.

\subsection{GSH Design}

Like LTE Rel-8. Rel-10 also use high-level signaling to decide open or close slot level of GSH function, and define the GSH in two ways, one is slot level of GSH, one is subframe level of GSH, switching between two ways through high-level decision slot level of GSH opening or closing [10]. That is, if enable GSH, slot level of GSH is used at this time, if GSH disable, a sub-frame level of GSH is used, not off GSH function, this can be based on whether it is equal bandwidth UE pairing, by opening and closing GSH to select different GSH methods. If MU-MIMO UE is equal bandwidth resource allocation, Rel-8, 9, 10 are same, use slot level of GSH and can use the same way hopping. If unequal bandwidth paired Rel-10 UEs, use sub-frame level of GSH, two slots using the same 
sequence or a sequence group. If unequal bandwidth of Rel-8 or Rel-9 UEs with Rel-10 UEs pairs then closed (disable) GSH. No slot or sub-frame level of GSH for UEs in Rel-8 or Rel-9, for UE in Rel-10 use sub-frame level of GSH. UEs in Rel-8 or Rel-9 lost randomized performance of inter-cell interference, but the impact on the system as a whole should not be very large.

\subsection{CS, OCC and GSH Joint Design}

Through the above design and analysis, we give the following CS, OCC and GSH joint DMRS design method. As shown in Table 4. If an unequally bandwidth UEs pairing exists, with layer of greater than 2 layers, GSH is turned off, with numbered 4, 5, 6, 7 scheme (shaded area) to realize the sub-frame level of GSH. Other equal bandwidth paired UE, or when the layer is less than 2, you can use all the way [11].

Table 4. CS and the OCC Design Structure

\begin{tabular}{|c|c|c|c|c|c|c|c|c|}
\hline \multirow{2}{*}{ Number } & \multicolumn{5}{|c|}{ CS Index } & \multicolumn{5}{c|}{ OCC pattern } \\
\cline { 2 - 9 } & Layer 1 & Layer 2 & Layer 3 & Layer 4 & Layer 1 & Layer 2 & Layer 3 & Layer 4 \\
\hline 0 & 0 & 6 & 3 & 9 & 0 & 0 & 1 & 1 \\
\hline 1 & 2 & 8 & 5 & 11 & 1 & 1 & 0 & 0 \\
\hline 2 & 3 & 9 & 0 & 6 & 1 & 1 & 0 & 0 \\
\hline 3 & 4 & 10 & 7 & 1 & 0 & 0 & 1 & 1 \\
\hline 4 & 6 & 0 & 9 & 3 & 1 & 1 & 1 & 1 \\
\hline 5 & 8 & 2 & 11 & 5 & 0 & 0 & 0 & 0 \\
\hline 6 & 9 & 3 & 6 & 0 & 0 & 0 & 0 & 0 \\
\hline 7 & 10 & 4 & 1 & 7 & 1 & 1 & 1 & 1 \\
\hline
\end{tabular}

\subsection{Application Scenarios}

SU-MIMO design is relatively simple, here mainly introduce the equal bandwidth and unequal bandwidth matched MU-MIMO design.

Scenario 1: Rel-10 2-UEs, each UE using 4-layer SU-MIMO, equal bandwidth allocation.

Table 5.1. Rel-10 2-UEs Equal Bandwidth Resource Allocation

\begin{tabular}{|c|c|c|c|c|c|c|c|c|}
\hline UE & CS1 & CS2 & CS3 & CS4 & OCC1 & OCC2 & OCC3 & OCC4 \\
\hline UE1 & 0 & 6 & 3 & 9 & 0 & 0 & 1 & 1 \\
\hline UE2 & 4 & 10 & 7 & 1 & 0 & 0 & 1 & 1 \\
\hline
\end{tabular}

Using the inter slot of the GSH, as shown in Table 5.1. We can see that this design method can keep the maximum interval between each user's own layers.

Scenario 2: Composed of Rel-10 2-UEs/4-layer MU-MIMO, unequal bandwidth allocation.

Table 5.2. Rel-10 2-UEs Unequal Bandwidth Resource Allocation

\begin{tabular}{|c|c|c|c|c|c|c|c|c|}
\hline UE & CS1 & CS2 & CS3 & CS4 & OCC1 & OCC2 & OCC3 & OCC4 \\
\hline UE1 & 10 & 4 & 1 & 7 & 1 & 1 & 1 & 1 \\
\hline UE2 & 9 & 3 & 6 & 0 & 0 & 0 & 0 & 0 \\
\hline
\end{tabular}

Using inter sub-frame of GSH, as shown in Table 5.2, CS interval of each user are 3, so as to maintain the maximum interval between layers, the user through the different OCC to realize orthogonal.

Scenario 3: LTE-Advanced 2-UEs, each UE use 2-layer SU-MIMO, LTE-Advanced 1-UE use 4-layer SU-MIMO, unequal bandwidth allocation. 
Table 5.3. UE1/2-layer, UE2/2-layer and UE3/4-layer Unequal Bandwidth Allocation

\begin{tabular}{|c|c|c|c|c|c|c|c|c|}
\hline UE & CS1 & CS2 & CS3 & CS4 & OCC1 & OCC2 & OCC3 & OCC4 \\
\hline UE1 & 0 & 6 & -- & -- & 0 & 0 & -- & -- \\
\hline UE2 & 4 & 10 & -- & -- & 0 & 0 & -- & -- \\
\hline UE3 & 6 & 0 & 9 & 3 & 1 & 1 & 1 & 1 \\
\hline
\end{tabular}

Unequal bandwidth using inter sub-frames of GSH, as shown in Table 5.3, Because UE1 and UE2 are of equal bandwidth, so the use of the same OCC value of 0 . They and UE3 are unequal bandwidth pairing, UE3 OCC value is 1, use inter sub-frame of GSH can ensure better orthogonality between UE and here also can select other CS value, use more flexible.

Scenario 4: Composed of Rel-8 2-UEs/1-layer and Rel-10 2-UEs/2-layer MU-MIMO, equal bandwidth allocation.

Table 5.4. Rel-8 2-UEs/1-layer, Rel-8 2-UEs/2-layer Equal Bandwidth Allocation

\begin{tabular}{|c|c|c|c|c|}
\hline UE & CS1 & CS2 & OCC1 & OCC2 \\
\hline UE1(R8) & 0 & -- & 0 & -- \\
\hline UE2(R8) & 6 & -- & 1 & -- \\
\hline UE3(R10) & 2 & 8 & 1 & 1 \\
\hline UE4(R10) & 4 & 10 & 0 & 0 \\
\hline
\end{tabular}

Equal bandwidth of the MU-MIMO, OCC not for orthogonal effect between layers, so design is more flexible, with the inter slot of GSH, as shown in Table 5.4.

\section{Simulation and Performance Analysis}

First simulation environment and the simulation parameters are given, as shown in Table 6.

Table 6. Simulation Parameters

\begin{tabular}{|c|c|}
\hline simulation parameter & Value \\
\hline Carrier frequency & $2 \mathrm{GHz}$ \\
\hline Bandwidth & $10 \mathrm{MHz}$ \\
\hline CP length & 72 \\
\hline FFT size & 1024 \\
\hline MIMO allocation & $2 \mathrm{~T}^{\times} 2 \mathrm{R}, 3 \mathrm{~T}^{\times} \times 3 \mathrm{R}, 4 \mathrm{~T} \times 4 \mathrm{R}$ \\
\hline Channel model & $\mathrm{SCME}$ \\
\hline Physical resource blocks & $6 \mathrm{PRB}$ \\
\hline Movement speed & $3 \mathrm{Km} / \mathrm{h}$ \\
\hline Pilot structure & Only CDM CS, Combining CS and OCC \\
\hline
\end{tabular}

Simulation 1: Performance comparison of only CS and CS-OCC combining

Introduce OCC in DMRS design, hoping to bring some performance gains. Compares using only CS and CS-OCC combining performance in two ways, as shown in Figure 4. 


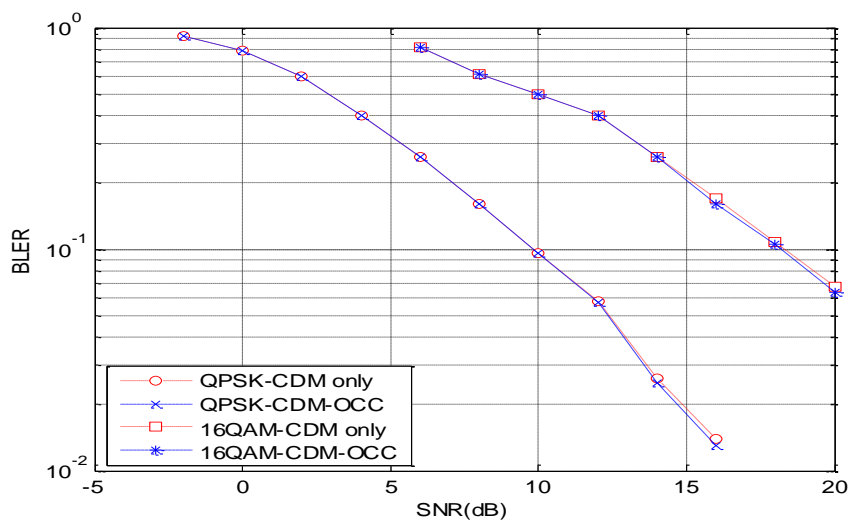

\section{Figure 4. Comparison of Only CS and CS-OCC Combined with BLER Performance}

The simulation results show that, OCC introduced to improve the performance is very limited, so often take OCC as an assistant orthogonal method, introduce OCC can support different bandwidth user pairing, which is an important reason for the introduction of OCC. Therefore, in the LTE-Advanced system, using CS and OCC combination.

Simulation 2: Performance comparison of SU-MIMO different pilot design in LTEAdvanced

Following through the simulation and comparison of different design solutions, select a suitable pilot pattern. Figure 5 and 6, respectively is the LTE-Advanced uplink 2T2R, 4T4R BLER performance comparison of different pilot structures.

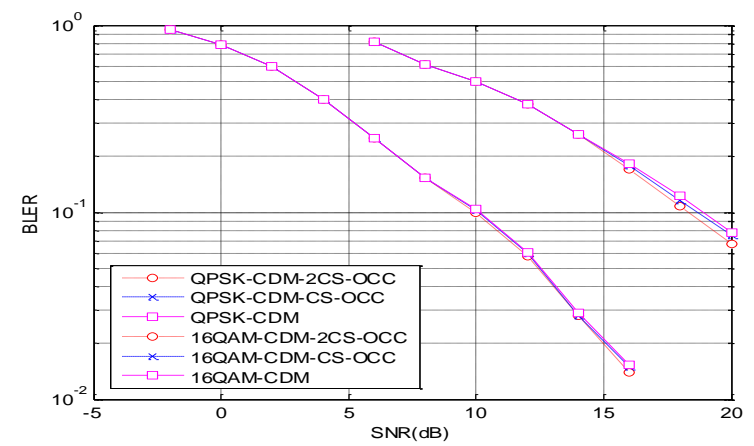

Figure 5. LTE-Advanced 2T $\times 2$ R BLER Performance

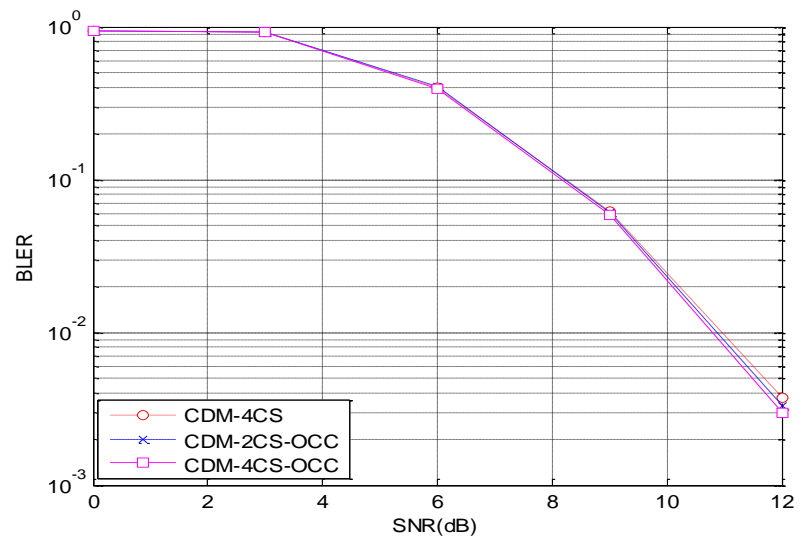

Figure 6. LTE-Advanced 4T $\times 4 R$ BLER Performance 
The simulation results show that, both QPSK and 16QAM modulation using OCC can bring some performance gains. OCC as a complementary orthogonal performance was better than OCC as an orthogonal, it is because the use of OCC as a main orthogonal way, when channel estimation two slots must be estimated jointly, namely two slot together can only estimate a channel, and OCC as the supplementary way each slot can estimate a channel, so the estimation accuracy is better than only using OCC. Between different pilot sequence are orthogonal, and OCC are completely orthogonal, so CS combined with OCC of best performance. To sum up, in the LTE-Advanced system, we select CDM-2CS-OCC and CDM-4CS-OCC as the pilot scheme of the 2 antenna and the 4 antenna.

Simulation 3: LTE-Advanced CS offset selection in the uplink DMRS 3-layer transmission

3-layer transmission in LTE-Advanced uplink CS offset has two options, as shown Table 1. The CS offset is 3, you can select CS value is $0,3,6$, CS offset is 4, you can choose CS value of $0,4,8$. We want CS intervals as large as possible, so that smaller the correlation between sequences, interfere with each other is smaller, but CS interval selection should also consider signaling design and compatibility. Simulation under the two ways, comparison of mean square error and block error rate performance, as shown in Figure 7 and 8, respectively is the 3-layer under different CS offset, MSE and BLER performance comparison.

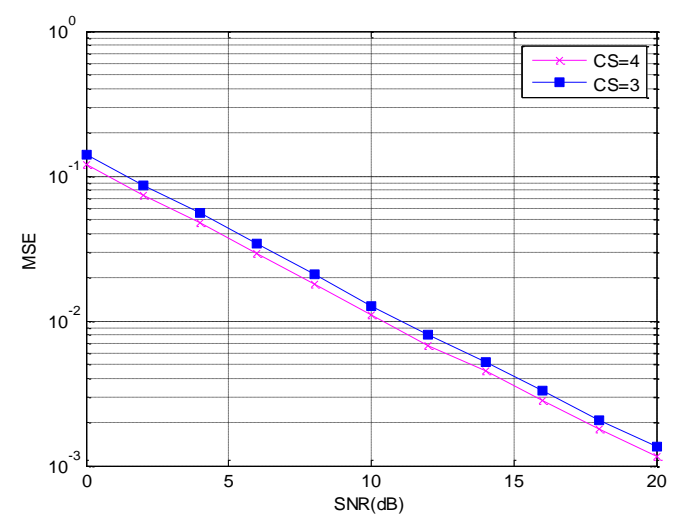

Figure 7. LTE-Advanced System of 3-layer Transmission under Different CS Offset MSE Performance

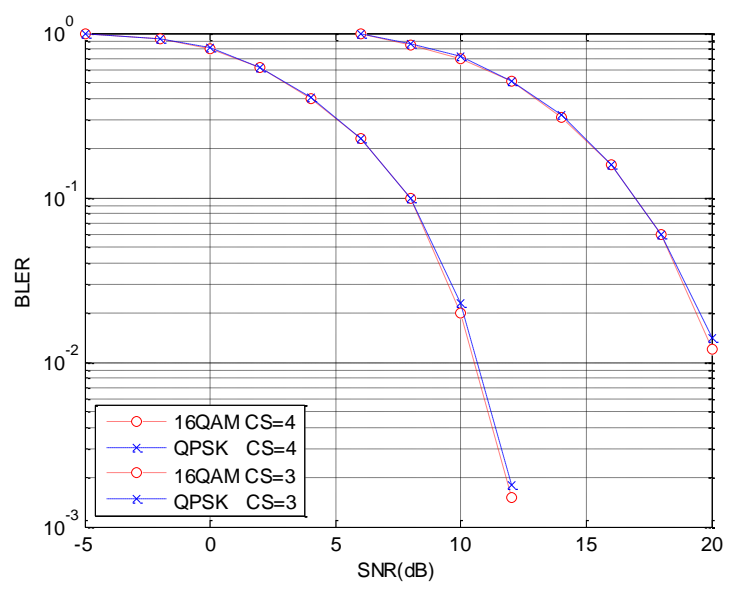

Figure 8. LTE-Advanced System of 3-layer Transmission under Different CS Offset BLER Performance 
The MSE results show, CS is 4 slightly better performance, than CS is 3 in $0.5 \sim 1 \mathrm{~dB}$ performance gains. Seen from the BLER performance, QPSK and 16QAM modulation, two ways performance differences are small. CS is 3 or 4 has little impact on system performance, taking into account the high level to low level of compatibility and signal design simplicity, we choose the interval of 3 .

\title{
5. Conclusion
}

By analyzing the LTE-Advanced DMRS existing problems in design, mainly related to $\mathrm{CS}, \mathrm{OCC}, \mathrm{GSH}$ signaling instructions and implementation issues. This paper proposes a CS、OCC and GSH joint instructions method of pilot design. Finally, simulation and comparison analysis of this scheme. The simulation results show that the improved design can satisfy the LTE-Advanced system well on the uplink DMRS requirements.

\section{ACKNOWLEDGEMENTS}

This work was supported by Major National Science and Technology Projects (No. 2011ZX03001-003-01) and the Sciences Youth Funded Projects for Chongqing University of Posts and Telecommunications (No.A2012-89). The authors would like to thank the anonymous referees whose insightful comments helped us to improve the presentation of the paper. Meanwhile, express the same thanks to Dr. Li Xiaowen for all his kindness and help.

\section{References}

[1] Y. Jeong, M. Kim, M. Y. Chung, et al., "Frequency-Domain Packet Scheduling for Low PAPR in 3GPP LTE Uplink", International Journal of Smart Home, vol. 7, no. 1, (2013).

[2] A. Z. Yonis, M. F. L. Abdullah and M. F. Ghanim, "Effective Carrier Aggregation on the LTEAdvanced Systems", International Journal of Advanced Science and Technology, SERSC, vol. 41, (2012), pp. 15-26.

[3] Y. Rui, P. Cheng, M. Li, et al., "Carrier aggregation for LTE-advanced: uplink multiple access and transmission enhancement features", Wireless Communications, IEEE, vol. 20, no. 4, (2013).

[4] “3GPP R1-121954”, Remaining details of PUSCH DMRS for UL CoMP. Huawei, (2012) May 21-25.

[5] “3GPP R1-122144”, Consideration on uplink DMRS issues for UL CoMP.ST-Ericsson, Ericsson, (2012) May 21-25.

[6] G. Lili, Z. Xin, L. Jihua, "Walsh yards of spectrum analysis", Harbin engineering Journal of learning, vol. 24, no. 5, (2003) October.

[7] “3GPP R1-102877”, OCC and CS for UL DMRS in SU/MU-MIMO, Panasonic, (2010) May 10-14.

[8] X. Hou and H. Kayama, "Demodulation reference signal design and channel estimation for LTEAdvanced uplink", INTECH Open Access Publisher, (2011).

[9] “3GPP R1-102141”, OCC and Uplink DM-RS for LTE-A, Motorola, (2010) April 12-16.

[10] Y. H. Nam, Y. Akimoto, Y. Kim, et al., "Evolution of reference signals for LTE-Advanced systems", Communications Magazine, IEEE, vol. 50, no. 2, (2012), pp. 132-138.

[11] "3GPP R1-102964", OCC configuration and sequence group hopping", Nokia Siemens Networks, Nokia, (2010) May 10-14.

\begin{abstract}
Authors
Xiaowen Li, received his M.S. degree in Electrical Engineering from Chongqing University, Chongqing, China in 1988. He is currently with the School of Communication and Information Engineering at Chongqing University of Posts and Telecommunications, China, as a Professor and Director of the Institute of Mobile communication protocol. His research interests
\end{abstract}


include wireless communications and MIMO. He has published more than 60 papers in journals and 30 papers in international conferences.

Youbo Feng, received the B.S. degree from Chongqing University of Posts and Telecommunications, Chongqing, China, in 2013. He is a Postgraduate Student of Chongqing University of Posts and Telecommunications, China, and will receive his M.S. degree in Communication and Information Systems in 2016. His research interests include LTE-Advanced physical layer technology, MIMO communications, and digital signal processing.

Dan Wang, was born in China in 1981. She received her Ph.D. degree in communications and information engineering from Chongqing University, Chongqing, China, in 2014. Received the M.S. degree in circuits and system from Chongqing University, Chongqing, China, in 2006. From 2006 to 2009, she joined Chongqing Chongyou information technology. She has worked on a wide array of wireless communication technologies including TD-SCDMA, GSM, and LTE. Since 2009, she has been an engineer in communication engineering, Chongqing University of Posts and Telecommunications and researching on LTE physical layer technology now. 
International Journal of Smart Home

Vol. 9, No. 4 (2015) 